\title{
Comparative Study on the Synergistic Action of Differentially Synthesized Silver Nanoparticles with $\beta$-Cephem Antibiotics and Chloramphenicol
}

\author{
Neethu Hari, ${ }^{1}$ Tincy K. Thomas, ${ }^{2}$ and A. Jayakumaran Nair ${ }^{2}$ \\ ${ }^{1}$ Centre for Nanoscience and Nanotechnology, University of Kerala, Kariavattom Campus, Thiruvananthapuram, Kerala 695581, India \\ ${ }^{2}$ Department of Biotechnology, University of Kerala, Kariavattom Campus, Thiruvananthapuram, Kerala 695581, India
}

Correspondence should be addressed to A. Jayakumaran Nair; ajayakumarannair@gmail.com

Received 31 July 2014; Revised 27 October 2014; Accepted 27 October 2014; Published 13 November 2014

Academic Editor: Mingwang Shao

Copyright (c) 2014 Neethu Hari et al. This is an open access article distributed under the Creative Commons Attribution License, which permits unrestricted use, distribution, and reproduction in any medium, provided the original work is properly cited.

Synergistic activity of cephem antibiotics with silver nanoparticles (Ag NPs) was investigated. Silver nanoparticles were synthesized through biological and chemical method. The combined action of $\beta$-lactam cephem antibiotics with both green and chemically synthesized silver nanoparticles enhances the antibacterial activity against wide range of antibiotic resistant pathogens and making them applicable to medical devices and microbial control systems. Synergistic activity of chloramphenicol with silver nanoparticles was also studied.

\section{Introduction}

Beta-lactam antibiotics (penams and cephems) are the largest group of antibiotics widely used in clinical medicine because of their high antibacterial activity [1]. $\beta$-Lactam groups of compounds are the most successful example of natural product application and chemotherapy [2]. All the natural and semisynthetic derivatives of $\beta$-lactam have their own properties and wide range of applications. Cephem derivatives are one of the new groups of $\beta$-lactam antibiotics with cephem ring and they show potent activity against different types of bacteria. Cephem class includes cephalosporins and cephamycins of which more than 22 antibiotics are now in clinical use and the efficacies of these drugs depend on their antibacterial spectra, potencies, and concentrations in tissues. But the empirical therapy and extent use of these agents have caused the development of bacterial resistance by different mechanisms like production of different kinds of $\beta$-lactamases ( $\beta$-lases), alteration of the antibiotic target site, and prevention of access of the antibiotic to the target by way of altered permeability or forced efflux and this may compromise the potential of $\beta$ lactam antibiotics. In this regard, considerable attention has been given to silver nanoparticles (Ag NPs), which have well-known antimicrobial properties and are used extensively in various medical and general applications and also the antibacterial, antifungal, and antiviral properties of silver ions, silver compounds, and silver nanoparticles have been extensively studied [3]. Moreover, high surface to volume ratio of silver nanoparticles may contribute to their enhanced antimicrobial properties by increasing the contact surface of silver nanoparticles with the microorganisms. Several studies also suggest that silver nanoparticles, which are well known to have broad spectrum antimicrobial activity against bacteria, virus, and eukaryotic microorganisms [4], interact with the cell membrane and some of them will penetrate the bacterial cell wall, thereby causing the death of bacteria $[5,6]$ Synergistic action is now commonly used to describe an interaction of two antimicrobial agents or occasionally more than two [7], in which the effect produced by the drugs in combination is greater than the sum of their individual effects when the antibiotics are used alone [8]. In another study, Vivekanandan et al. proposed that the silver nanoparticles have the ability to destroy the stability of lipopolysacchrides allowing increase in permeability of outer membrane and 
the peptidoglycan structure, which was recognized and captured by antibiotics (e.g., cephalexin) immediately, and the conjugation of antibiotics with silver nanoparticles makes the resistant strain to become sensitive to cephalexin [9]. This same mechanism was also possible for the synergistic study of cephem antibiotics (cefazolin and cephalothin) with silver nanoparticles. In this study, we investigated the synergistic activity of cephems (cefazolin and cephalothin) and chloramphenicol alone and also in combination with biogenic (AS-Ag NPs) and chemically synthesized (CS-Ag NPs) silver nanoparticles against different pathogens. It was found that, compared to the effects of silver nanoparticles and antibiotics alone, the combined effect of both of them was more pronounced, which suggests synergistic action of the two components. Minimum inhibitory concentration (MIC) was determined to confirm the antibacterial susceptibility and synergistic effects. The results emphasize that Ag NPs possessed antibacterial effects and synergistic activities.

\section{Materials and Methods}

2.1. Synthesis of Silver Nanoparticles. Silver nanoparticles were biologically synthesized using aqueous extracts of Allium sativum (AS-Ag NPs) and this extract was used as reducing and capping agent which was purchased from local market, while in chemical synthesis sodium citrate was used as capping and reducing agent. Silver nitrate $\left(\mathrm{AgNO}_{3}\right)$ was used as the precursor, which was from Merck (Mumbai, India).

2.2. Biogenic Synthesis of Silver Nanoparticles. For the synthesis of silver nanoparticles, equal volumes of pale white coloured garlic extracts were added to an equal quantity of $0.1 \mathrm{M}$ aqueous silver nitrate $\left(\mathrm{AgNO}_{3}\right)(1: 1$ ratio) solution by volume at room temperature. Here the $\mathrm{pH}$ of the extract was adjusted to alkaline condition before adding the silver nitrate solution. The reaction was carried out under dark condition to reduce the effects of photo activation. The reactants were then characterized at intervals to detect the presence of silver nanoparticles. The percentage of silver nanoparticles in the reaction medium was enhanced by three cycles of centrifugation at 10,000 rpm for $5 \mathrm{~min}$, followed by redispersion in deionized water.

2.3. Chemical Synthesis of Silver Nanoparticles. The chemical synthesis of silver nanoparticle was done using Lee and Meisel's method [10]. Silver nitrate solution was prepared by dissolving $90 \mathrm{mg}$ of silver nitrate in $500 \mathrm{~mL}$ of deionized water and solution was allowed to boil. Sodium citrate solution (10 $\mathrm{mL}$ of $1 \%$ solution) was added dropwise to the boiling solution with vigorous stirring and then the solution was kept boiling for a further period of 10 minutes, until a grey dispersion of silver nanoparticles resulted. The precipitate was separated by centrifugation, washed repeatedly using deionized water to remove any adsorbed impurities, and dried. The preparation and processing procedures were carried out in the dark. The nanoparticles so-formed were characterized using UV-visible spectrum analysis and SEM analysis.
2.4. Conjugation of Cephem Antibiotics and Chloramphenicol with Silver Nanoparticles. Disc diffusion method was used to assay the synergistic effect of $\beta$-lactam cephems with green synthesized and chemically synthesized silver nanoparticles for bactericidal activity against test strains (Bacillus subtilis, Staphylococcus aureus, Micrococcus luteus, Lactococcus lactis, Escherichia coli, Pseudomonas aeruginosa, Salmonella typhi, Klebsiella pneumoniae, and Proteus vulgaris) on Muller Hinton agar plates. Here the standard antibiotic discs were purchased from Himedia (Mumbai, India) and the inocula were prepared by diluting the overnight cultures with $0.9 \% \mathrm{NaCl}$ to a $0.5 \mathrm{McF}$ arland standard and were applied to the plates along with the standard and prepared discs containing differing amounts of Ag NPs with antibiotic. After incubation at $35^{\circ} \mathrm{C}$ for 24-48 hours, the zones of inhibition were measured [11].

\section{Results and Discussion}

In this work we successfully synthesized silver nanoparticles using garlic (Allium sativum) and sodium citrate, respectively. The formation and the stability of the silver nanoparticles so-obtained were investigated and it was noted that a yellow colour was developed in solution, which has been attributed to the excitation of surface plasmon resonance (SPR) in Ag NPs. This SPR was arising due to collective oscillation of free conduction electron induced by an interacting electromagnetic field [12]. Here the addition of Allium sativum extract to the $\mathrm{AgNO}_{3}$ aqueous solution results in the reduction of $\mathrm{Ag}^{+}$ into Ag NPs within 30 minutes. In the case of biogenic synthesis, the aqueous extract of Allium sativum consists of bioactive compounds such as the sulfur compounds, alliin (S-allylL-cysteine sulfoxide) and ajoene, saponins, flavonoids, and allixin, and the water soluble ingredients of Allium sativum extracts are responsible for bioreduction of $\mathrm{Ag}^{+}$ions and stabilization of green synthesized silver nanoparticles (Ag NPs). The UV absorption spectra of green synthesized and chemical synthesized silver nanoparticles had an absorption peak at $438 \mathrm{~nm}$ and $404 \mathrm{~nm}$, respectively, and the broad spectra indicate that silver nanoparticles were polydispersed. The optical spectrum of the green synthesized silver nanoparticles exhibits only one dominant absorption band around $438 \mathrm{~nm}$ which represents the formation of dipole plasmon resonance of spherical nanoparticles (Figure 2). Same result was also noticed in the chemically synthesized nanoparticles. The position of the SPR band in UV-Vis spectra depended on the particle shape, particle size and its interaction with the medium, local refractive index, and the extent of charge transfers between medium and particles [13]. The peaks at $404 \mathrm{~nm}$ (Figure 1) and $438 \mathrm{~nm}$ are in good agreement with the theoretical simulation of SPR using Mie's theory [14].

Crystalline nature of the silver nanoparticles synthesized through green and chemical method was confirmed by the peaks in the XRD pattern corresponding to (111), (200), and (220) planes (JCPDS file number 04-0783). Similar results were reported earlier in silver nanoparticles [15].

The average particle size of silver nanoparticles was calculated using Debye-Scherrer equation. From the Scherrer equation, the average crystallite size was found to be $14 \mathrm{~nm}$ and $12 \mathrm{~nm}$ for AS-Ag NPs and CS-Ag NPs, respectively. A 


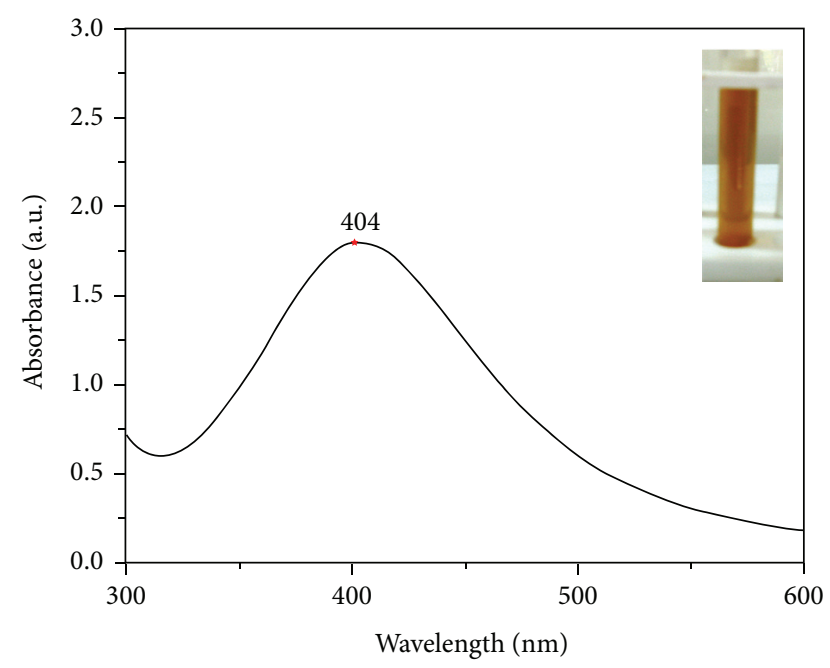

FIGURE 1: UV-visible spectrophotometer analysis of chemically synthesized silver nanoparticles.

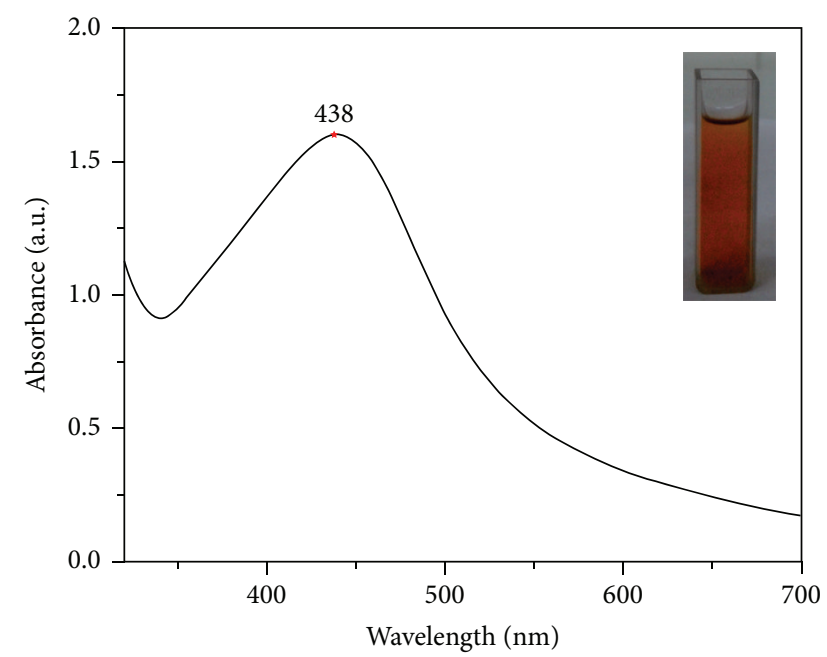

FIGURE 2: UV-visible spectrophotometer analysis of green synthesized silver nanoparticles.

comparison of XRD spectrum of CS-Ag NPs (Figure 4) with AS-Ag NPs showed some additional unassigned peaks in the XRD of AS-Ag NPs. The additional peak on XRD spectrum of AS-Ag NPs (Figure 3) may be due to the extract, which was used for the synthesis of silver nanoparticles which forms the capping for silver nanoparticles. Similar results were reported in silver nanoparticles synthesized using Murraya koenigii [16].

The SEM micrographs of these nanoparticles showed that the nanoparticles formed are roughly spherical nanoparticles and they are polydispersed (Figure 5). The combination of Ag NPs (AS-Ag NPs and CS-Ag NPs) with two different $\beta$-lactam cephem antibiotics (cefazolin and cephalothin) was tested against four gram-positive (Bacillus subtilis, Staphylococcus aureus, Micrococcus luteus, and Lactococcus lactis) and five gram-negative (Escherichia coli, Pseudomonas aeruginosa,

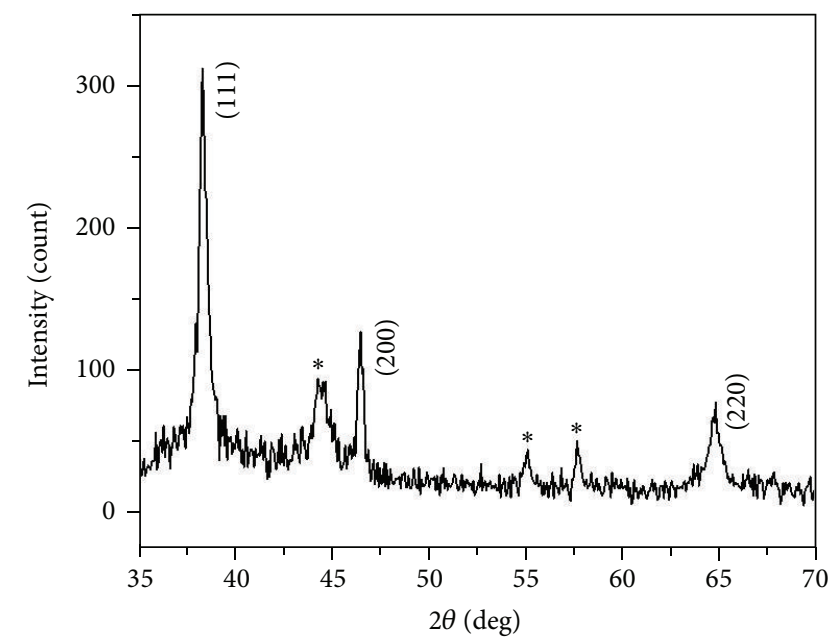

FIGURE 3: XRD of Ag NPs synthesized using Allium sativum ( ${ }^{*}$ shows unassigned peaks).

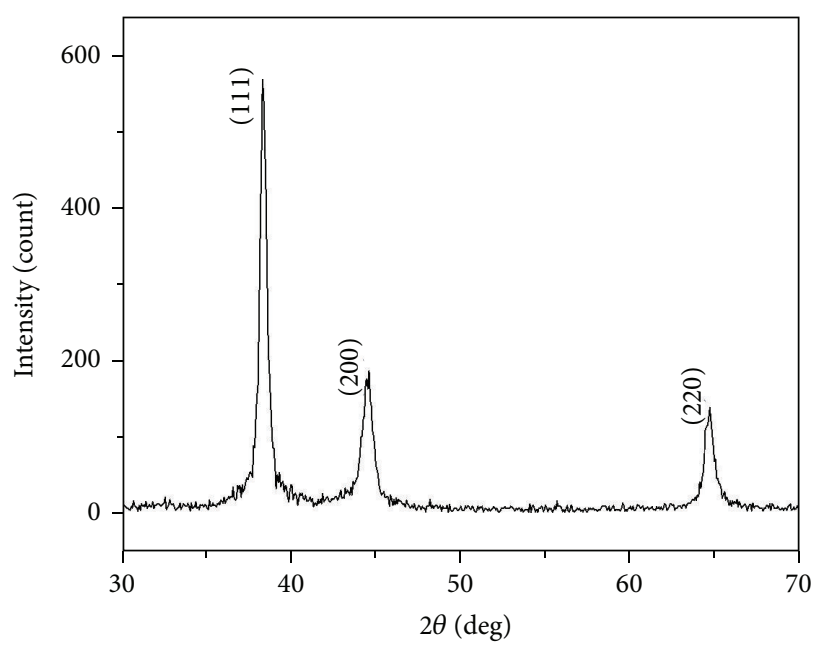

FIgURE 4: XRD of Ag NPs synthesized by chemical reduction method.

Salmonella typhi, Klebsiella pneumoniae, and Proteus vulgaris) bacteria using the disc diffusion method. The zone $(\mathrm{mm})$ of inhibition of different antibiotic discs with and without Ag NPs against test strains was measured. Both of the cephem antibiotics (cephalothin and cefazolin) showed a maximum fold increase of $30 \%$ antibacterial activity in combination with $20 \mu \mathrm{g} / \mathrm{mL}$ of AS-Ag NPs against Bacillus subtilis (Figure 7) and Micrococcus luteus (Figure 6), respectively.

In the case of cefazolin, chemically synthesized silver nanoparticles $20 \mu \mathrm{g} / \mathrm{mL}$ of Allium sativum together with cefazolin shows $15.3 \%$ fold increase against Pseudomonas aeruginosa, $10 \%$ fold increase against Micrococcus luteus, $7.1 \%$ fold increase against Lactococcus lactis, and 3.4\% and 3.2\% fold increase against Escherichia coli and Proteus vulgaris, respectively (Table 1), while cephalothin also showed an enhanced synergistic activity in the presence of AS-Ag NPs against different pathogens. It shows $18.8 \%, 9 \%$, and $5.8 \%$ fold increase against Micrococcus luteus, Staphylococcus aureus, 
TABLE 1: Synergistic activity of cefazolin against different pathogens.

\begin{tabular}{|c|c|c|c|c|c|}
\hline \multirow[b]{2}{*}{ Microorganisms } & \multirow{2}{*}{$\begin{array}{l}\text { Cefazolin } \\
\text { zone }(\mathrm{mm})\end{array}$} & \multicolumn{4}{|c|}{ Ag NPs + cefazolin } \\
\hline & & $\begin{array}{c}\text { Ag NPs (AS) } \\
30 \mu \mathrm{g} / \mathrm{disc}\end{array}$ & Fold increase (\%) & $\begin{array}{c}\text { Ag NPs (CS) } \\
20 \mu \mathrm{g} / \text { disc }\end{array}$ & Fold increase (\%) \\
\hline Escherichia coli & 29 & 26 & -10.3 & 30 & 3.4 \\
\hline Pseudomonas aeruginosa & 13 & 13 & 0 & 15 & 15.3 \\
\hline Staphylococcus aureus & 13 & 11 & -15.3 & 13 & 0 \\
\hline Lactococcus lactis & 14 & 16 & 14.28 & 15 & 7.1 \\
\hline Micrococcus luteus & 10 & 13 & 30 & 11 & 10 \\
\hline Bacillus subtilis & 27 & 27 & 0 & 24 & -11.1 \\
\hline Proteus vulgaris & 31 & 29 & -6.45 & 32 & 3.2 \\
\hline Klebsiella pneumoniae & 8 & 0 & -100 & 0 & -100 \\
\hline Salmonella typhi & 12 & 10 & -16.6 & 11 & -8.3 \\
\hline Proteus mirabilis & 18 & 17 & -5.5 & 17 & -5.5 \\
\hline
\end{tabular}

TABLE 2: Synergistic activity of cephalothin against different pathogens.

\begin{tabular}{|c|c|c|c|c|c|}
\hline \multirow[b]{2}{*}{ Microorganisms } & \multirow[b]{2}{*}{$\begin{array}{l}\text { Cephalothin } \\
\text { zone }(\mathrm{mm})\end{array}$} & \multicolumn{4}{|c|}{ Ag NPs + cephalothin } \\
\hline & & $\begin{array}{c}\text { Ag NPs (AS) } \\
30 \mu \mathrm{g} / \text { disc }\end{array}$ & Fold increase (\%) & $\begin{array}{c}\text { Ag NPs (CS) } \\
20 \mu \mathrm{g} / \text { disc }\end{array}$ & Fold increase (\%) \\
\hline Escherichia coli & 0 & 0 & 0 & 0 & 0 \\
\hline Pseudomonas aeruginosa & 14 & 10 & -28.5 & 15 & 7.1 \\
\hline Staphylococcus aureus & 11 & 12 & 9 & 10 & -9 \\
\hline Lactococcus lactis & 12 & 11 & -8.3 & 12 & 0 \\
\hline Micrococcus luteus & 11 & 13 & 18.18 & 10 & -9 \\
\hline Bacillus subtilis & 10 & 13 & 30 & 13 & 30 \\
\hline Proteus vulgaris & 34 & 22 & -35.2 & 24 & -29.4 \\
\hline Klebsiella pneumoniae & 0 & 0 & 0 & 0 & 0 \\
\hline Salmonella typhi & 13 & 13 & 0 & 14 & 7.6 \\
\hline Proteus mirabilis & 34 & 36 & 5.8 & 35 & 2.9 \\
\hline
\end{tabular}

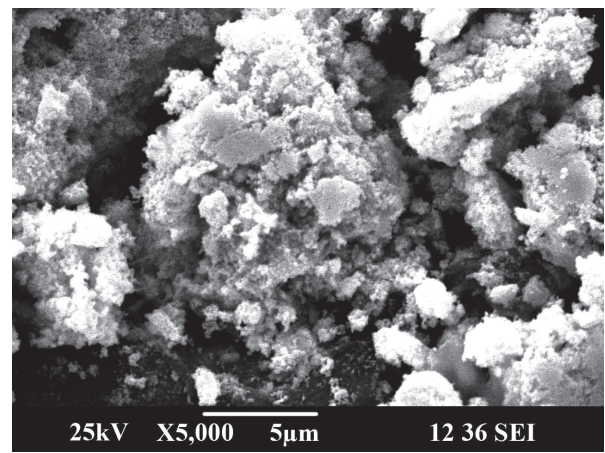

FIGURE 5: SEM image of silver nanoparticles.

and Proteus mirabilis, respectively, at a concentration of $20 \mu \mathrm{g} / \mathrm{mL}$ (Table 2), while CS-Ag NPs show 30\% (Figure 7), $7.6 \%, 7.1 \%$, and $2.9 \%$ fold increase against Bacillus subtilis, Salmonella typhi, Pseudomonas aeruginosa, and Proteus mirabilis, respectively (Table 2), at $20 \mu \mathrm{g} / \mathrm{mL}$. In the case of chemically synthesized Ag NPs, cephalothin showed the most pronounced antibiotic synergy against pathogens, while in biogenic synthesized silver nanoparticles both cephem antibiotics showed almost same synergistic activity.

The antibiotic chloramphenicol also showed high synergistic effect with both chemically and biogenically synthesized silver nanoparticles. It shows a maximum enhancement of $28.5 \%$ of fold increase for $20 \mu \mathrm{g} / \mathrm{mL}$ of AS-Ag NPs against Escherichia coli (Figure 8), and it also shows 25\%, 20\%, 16\%, 10.7\%, 3.57\%, and 3.3\% against Escherichia coli, Bacillus subtilis, Lactococcus lactis, Pseudomonas aeruginosa, Micrococcus luteus, Staphylococcus aureus, and Proteus vulgaris, respectively, for $20 \mu \mathrm{g} / \mathrm{mL}$ of AS-Ag NPs (Table 3). In the case of $20 \mu \mathrm{g} / \mathrm{mL}$ of CS-Ag NPs, chloramphenicol shows $25 \%, 10.7 \%$, 8\%, 7.1\%, 6.6\%, 5\%, 4.7\%, 4\%, and 3.5\% for Bacillus subtilis, Staphylococcus aureus, Pseudomonas aeruginosa, Escherichia coli, Proteus vulgaris, Lactococcus lactis, Proteus mirabilis, Salmonella typhi, and Micrococcus luteus, respectively. This study clearly indicates that combination of chloramphenicol with AS-Ag NPs also shows better synergistic activity. Moreover, the antibiotic activity increases with increase in concentration of silver nanoparticles. But it was observed in some cases that antibiotics in combination with Ag NPs (ASAg NPs and CS-Ag NPs) bring down the inhibitory effect of antibiotics activity. This type of antagonistic activity of 


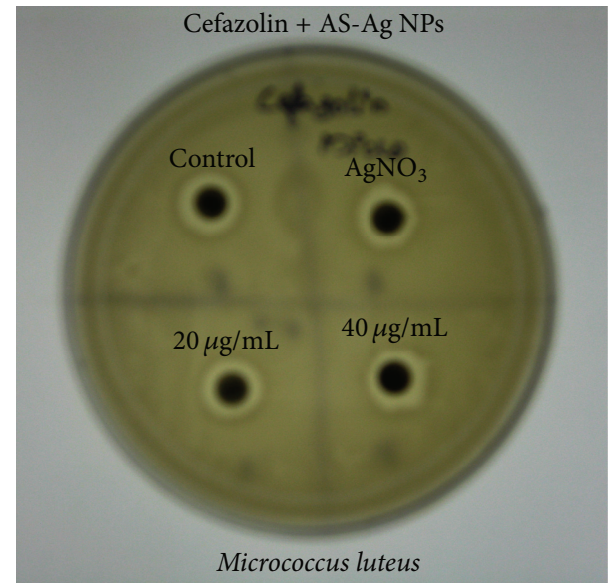

(a)

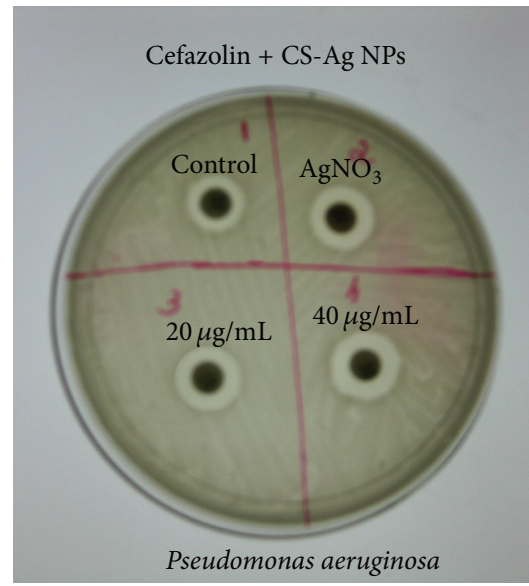

(b)

FIGURE 6: Synergistic activity of cefazolin with AS-Ag NPs and CS-Ag NPs against Micrococcus luteus and Pseudomonas aeruginosa.

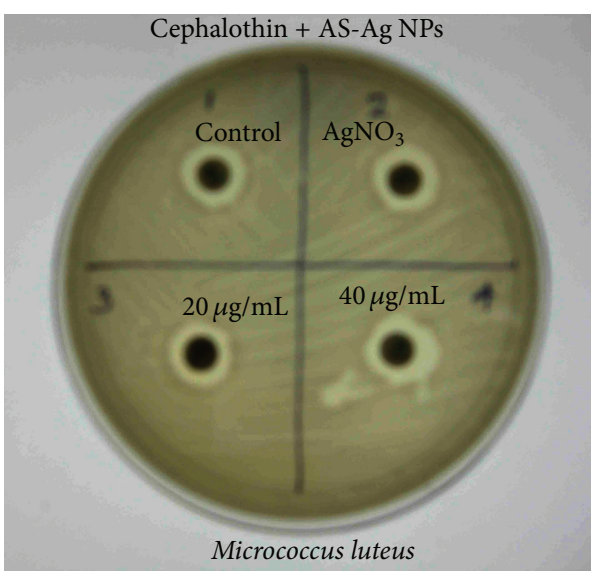

(a)

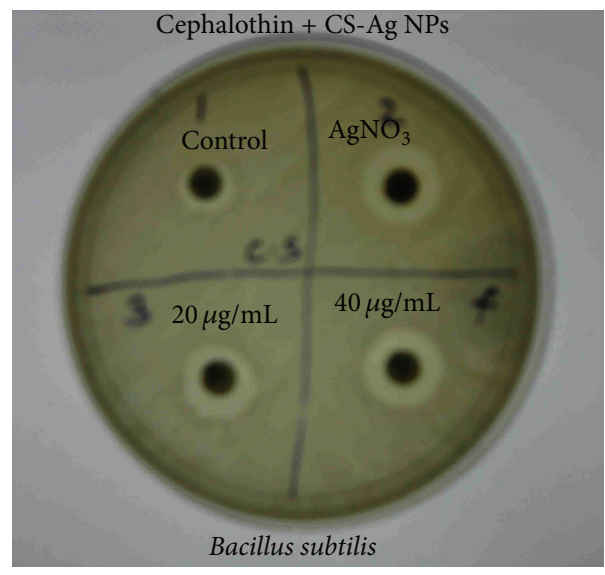

(b)

FIgURE 7: Synergistic activity of cephalothin with AS-Ag NPs and CS-Ag NPs against Micrococcus luteus and Bacillus subtilis.

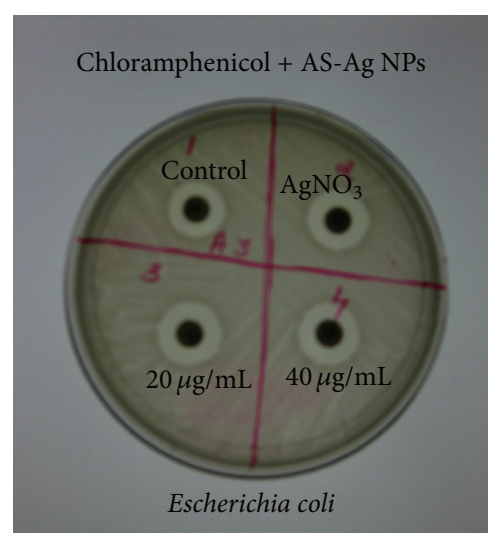

(a)

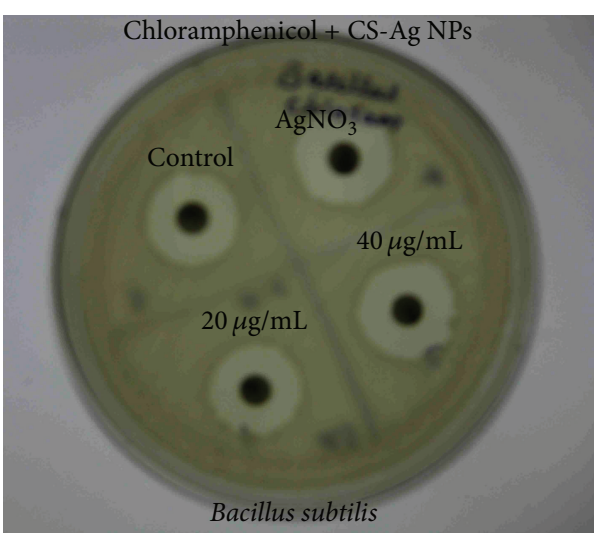

(b)

FIGURE 8: Synergistic activity of chloramphenicol with AS-Ag NPs and CS-Ag NPs against Escherichia coli and Bacillus subtilis. 
TABLE 3: Synergistic activity of chloramphenicol against different pathogens.

\begin{tabular}{|c|c|c|c|c|c|}
\hline \multirow[b]{2}{*}{ Microorganisms } & \multirow[b]{2}{*}{$\begin{array}{l}\text { Chloramphenicol } \\
\text { zone }(\mathrm{mm})\end{array}$} & \multicolumn{4}{|c|}{ Ag NPs + chloramphenicol } \\
\hline & & $\begin{array}{c}\text { Ag NPs (AS) } \\
30 \mu \mathrm{g} / \text { disc }\end{array}$ & Fold increase (\%) & $\begin{array}{c}\text { Ag NPs (CS) } \\
20 \mu \mathrm{g} / \mathrm{disc}\end{array}$ & Fold increase (\%) \\
\hline Escherichia coli & 28 & 36 & 28.5 & 30 & 7.1 \\
\hline Pseudomonas aeruginosa & 25 & 29 & 16 & 27 & 8 \\
\hline Staphylococcus aureus & 28 & 29 & 3.57 & 31 & 10.7 \\
\hline Lactococcus lactis & 20 & 24 & 20 & 21 & 5 \\
\hline Micrococcus luteus & 28 & 31 & 10.7 & 29 & 3.5 \\
\hline Bacillus subtilis & 16 & 20 & 25 & 20 & 25 \\
\hline Proteus vulgaris & 30 & 31 & 3.33 & 32 & 6.6 \\
\hline Klebsiella pneumoniae & 15 & 7 & -53.3 & 13 & -13.3 \\
\hline Salmonella typhi & 25 & 25 & 0 & 26 & 4 \\
\hline Proteus mirabilis & 21 & 21 & 0 & 22 & 4.7 \\
\hline
\end{tabular}

silver nanoparticles when combined with antibiotics is not reported so far. Both silver nanoparticles (AS-Ag NPs and CS-Ag NPs) showed this type of antagonistic activity when combined with some of the antibiotics. So these Ag NPs can be recommended together with antibiotics and without antibiotics in different cases as per the observed results for controlling the pathogens.

In a previous investigation it was showed that Ag NPs with high surface to volume ratio can easily attach to the cell membrane. The interaction between nanoparticles and the cell membrane will disturb the function of cell such as permeability, respiration, and so forth and eventually lead to cell death. The antibacterial activity of silver nanoparticles has also been related to the action of the ion released from them [17], while in the study of Kim et al., they reported that the antimicrobial effect of silver nanoparticle is mediated by the generation of free radicals [18]. Some reports showed the affinity of silver nanoparticles to react with phosphorus and sulphur compounds. Since the membrane of the bacteria contains many sulphur-containing proteins, these might be preferential sites for the silver nanoparticles. On the other hand, nanoparticles inside the bacteria will also tend to react with other sulphur-containing proteins in the interior of the cell, as well as with phosphorus-containing compounds such as DNA, and result in bacterial death [19]. It has also been reported that the combination of $\mathrm{Ag}$ NPs and cinnamaldehyde showed near-synergistic mode of inhibition against various pathogenic and food spoilage bacteria [20].

Some hypothesis indicated that catalytic oxidation of silver ions with nascent oxygen reacts with bacterial cell membrane, leading to cell death. Batarseh reported that silver chelates prevent DNA from unwinding which may result in serious damage to cell death [21]. In another report penicillin $\mathrm{G}$, amoxicillin, erythromycin, clindamycin, and vancomycin in combination with the mycosynthesized Ag NPs against Escherichia coli, Pseudomonas aeruginosa, and Staphylococcuss aureus showed enhanced antibacterial activity. Fayaz et al. also reported an increase in the antibacterial activities of ampicillin, kanamycin, erythromycinm and chloramphenicol in combination with Ag NPs against Salmonella typhi, Escherichia coli, Staphylococcus aureusm and Micrococcus luteus [11]. Formulation of Ag NPs in combination with commonly used antibiotics can be used as effective bactericidal agents. In a previous study it was shown that the antibiotic chloramphenicol acts on the 50S subunit of ribosome in bacteria, inhibiting translation by blocking either the peptidyl transferase reaction or the translocation step [22].

Silver nanoparticles with high surface to volume ratio increase the chloramphenicol uptake by increasing the permeability of cell membranes. In a recent study, synergistic activity of triangular shaped silver nanoparticles along with antibiotics ampicillin and gentamicin was studied against Staphylococcus aureus and Pseudomonas aeruginosa. In that study triangular nanoprism with sharp vertices and edges displays higher antibacterial activity in comparison to other shapes of silver nanoparticles which finds these nanoparticles useful for biomedical applications [23]. Vivekanandan et al. was also reported the synergistic antibacterial activity of cephalexin with Ag NPs [9]. In our study nonhydrophilic nature of nanosilver facilitates the transport of antibiotics (cefazolin, cephalothin, and chloramphenicol) to the cell membrane which consists of phospholipids and glycoprotein (Figure 9) which are all hydrophobic groups and the synergistic effect may be caused by the bonding reaction between hydroxyl and amido groups of antibiotics and nanosilver; then the antibiotic-silver nanoparticle combination may attach on the cell membrane and result in the lysis of cell wall of bacteria, which was followed by the entry of Ag NPantibiotic combination into the cell and may result in the DNA unwinding and cell death (Figure 9). Some of the previous work also reported the mechanism of DNA unwinding by the attachment of ampicillin-Ag NP combination on bacterial cell [8]. The effect induced by the silver nanoparticles alone indicated that their antimicrobial effect is not as good as the combination of Ag NPs and antibiotics and the combination of silver nanoparticles are found to be effective at lower concentration. 


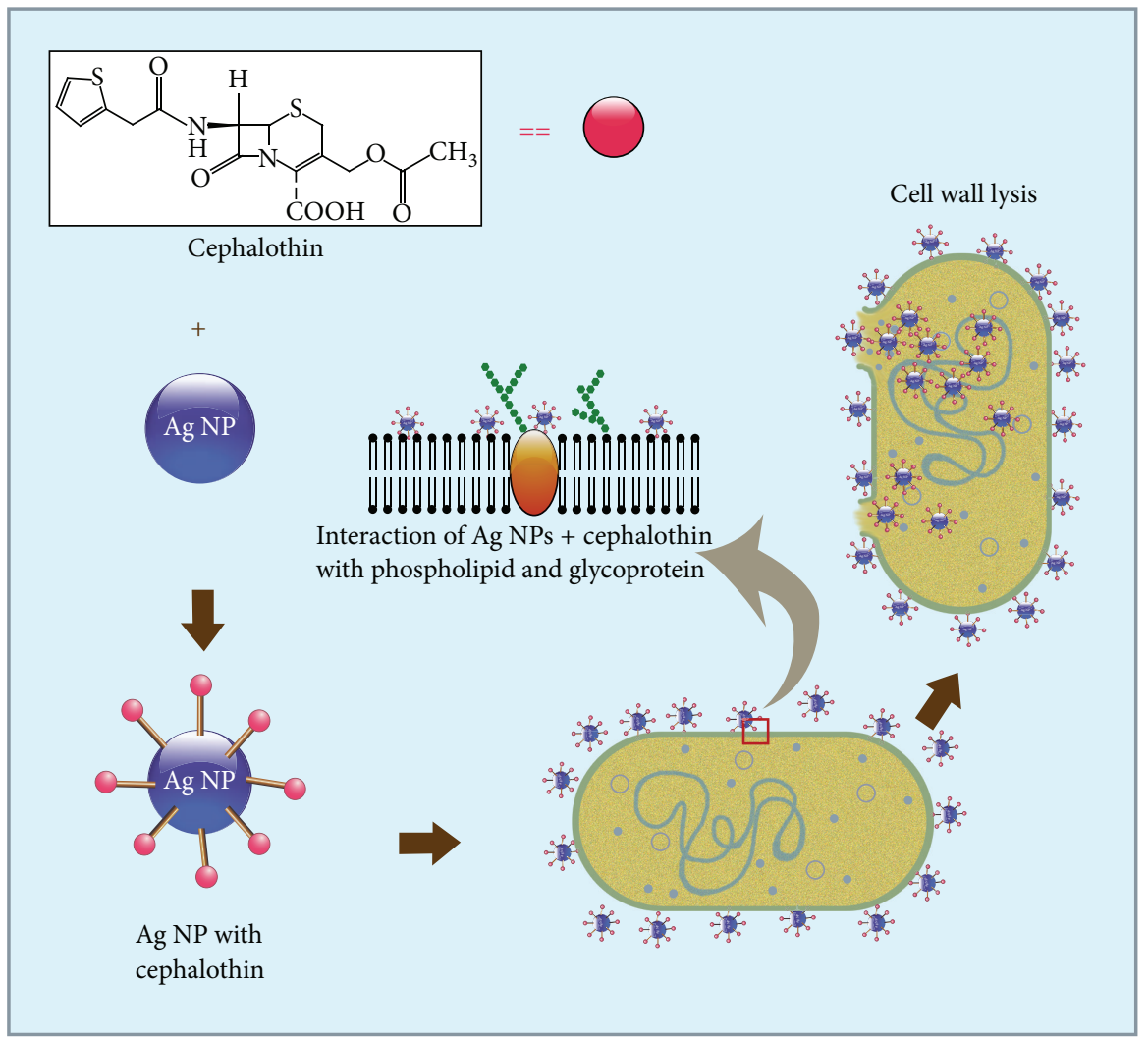

FIGURE 9: Synergistic action of antibiotic (cephalothin) conjugated silver nanoparticles against bacterial cell.

\section{Conclusion}

The study emphasized on a possible combination of $\beta$-lactam cephem antibiotics (cefazolin and cephalothin) and chloramphenicol with Allium sativum synthesized and citrate capped Ag NPs, which showed enhanced antimicrobial effects and was concluded as synergism. In this context, synergistic antibacterial property of silver nanoparticles with cephem antibiotics, cephalothin and cefazolin, is considered as an alternative and attractive method to combat the increasing spread of drug resistance and such an approach is likely to provide much potential application in medical devices and microbial resistant system. Since the cephem antibiotics are relatively costly comparing with other $\beta$-lactam antibiotics, in our study we reduce the concentration of cephem antibiotics for minimizing the cost and side effects by combining them with silver nanoparticles.

\section{Conflict of Interests}

The authors confirm that there are no known conflicts of interest associated with this publication and there has been no significant financial support for this work that could have influenced its outcome.

\section{Acknowledgment}

The authors are thankful to Dr. M. Abdul Khadar, Honorary Director, Centre for Nanoscience and Nanotechnology,
University of Kerala, for his suggestions, to NIIST \& Alagappa University for XRD analysis, and also to STIC, Cochin University, Kerala, for SEM.

\section{References}

[1] G. L. Mandel and A. M. Sande, "Penicillins, cephalosporins, and other beta-lactam antibiotics," in The Pharmacological Basis of Therapeutics, A. Goodman Gilman, T. W. Rall, A. S. Nies, and P. Taylor, Eds., pp. 1271-1291, Pergamon Press, New York, NY, USA, 8th edition, 1990.

[2] A. L. Demain and R. P. Blander, "The $\beta$-lactam antibiotics: past, present, and future," Antonie van Leeuwenhoek, vol. 75, no. 1-2, pp. 5-19, 1999.

[3] K. Ito, M. Hayasaki, and T. Tamaya, "Pharmacokinetics of cephem antibiotics in exudate of pelvic retroperitoneal space after radical hysterectomy and pelvic lymphadenectomy," Antimicrobial Agents and Chemotherapy, vol. 34, no. 6, pp. 11601164, 1990.

[4] Q. L. Feng, J. Wu, G. Q. Chen, F. Z. Cui, T. N. Kim, and J. O. Kim, "A mechanistic study of the antibacterial effect of silver ions on Escherichia coli and Staphylococcus aureus," Journal of Biomedical Materials Research, vol. 52, no. 4, pp. 662-668, 2000.

[5] I. Sondi and B. Salopek-Sondi, "Silver nanoparticles as antimicrobial agent: a case study on E. coli as a model for Gramnegative bacteria," Journal of Colloid and Interface Science, vol. 275, no. 1, pp. 177-182, 2004.

[6] L. F. Espinosa-Cristóbal, G. A. Martínez-Castañón, R. E. Martínez-Martínez, J. P. Loyola-Rodríguez, J. F. Reyes-Macías, 
and F. Ruiz, "Antibacterial effect of silver nanoparticles against Streptococcus mutans," Materials Letters, vol. 63, no. 29, pp. 2603-2606, 2009.

[7] M. C. Berenbaum, "A method for testing for synergy with any number of agents," The Journal of Infectious Diseases, vol. 137, no. 2, pp. 122-130, 1978.

[8] E. Jawetz, "The use of combinations of antimicrobial drugs," Annual Review of Pharmacology, vol. 8, pp. 151-170, 1968.

[9] K. E. Vivekanandan, K. G. Raj, S. Kumaresana, and M. Pandib, "Biosynthesis of silver nanoparticle activity against bacterial strain, cephalexin antibiotic synergistic activity," International Journal of Current Science, vol. 4, pp. 1-7, 2012.

[10] P. C. Lee and D. Meisel, "Adsorption and surface-enhanced Raman of dyes on silver and gold sols," The Journal of Physical Chemistry, vol. 86, no. 17, pp. 3391-3395, 1982.

[11] A. M. Fayaz, K. Balaji, M. Girilal, R. Yadav, P. T. Kalaichelvan, and R. Venketesan, "Biogenic synthesis of silver nanoparticles and their synergistic effect with antibiotics: a study against gram-positive and gram-negative bacteria," Nanomedicine: Nanotechnology, Biology, and Medicine, vol. 6, no. 1, pp. e103e109, 2010.

[12] P. Mulvaney, "Surface plasmon spectroscopy of nanosized metal particles," Langmuir, vol. 12, no. 3, pp. 788-800, 1996.

[13] V. K. Vidhu, S. A. Aromal, and D. Philip, "Green synthesis of silver nanoparticles using Macrotyloma uniflorum," Spectrochimica Acta Part A: Molecular and Biomolecular Spectroscopy, vol. 83, no. 1, pp. 392-397, 2011.

[14] S. Baset, H. Akbari, H. Zeynali, and M. Shafie, "Size measurement of metal and semiconductor nanoparticles via UVVis absorption spectra," Digest Journal of Nanomaterials and Biostructures, vol. 6, no. 2, pp. 709-716, 2011.

[15] V. K. Vidhu and D. Philip, "Catalytic degradation of organic dyes using biosynthesized silver nanoparticles," Micron, vol. 56, pp. 54-62, 2014.

[16] D. Philip, C. Unni, S. A. Aromal, and V. K. Vidhu, "Murraya Koenigii leaf-assisted rapid green synthesis of silver and gold nanoparticles," Spectrochimica Acta Part A: Molecular and Biomolecular Spectroscopy, vol. 78, no. 2, pp. 899-904, 2011.

[17] J. R. Morones, J. L. Elechiguerra, A. Camacho et al., "The bactericidal effect of silver nanoparticles," Nanotechnology, vol. 16, no. 10, pp. 2346-2353, 2005.

[18] J. S. Kim, E. Kuk, K. N. Yu et al., "Antimicrobial effects of silver nanoparticles," Nanomedicine: Nanotechnology, Biology, and Medicine, vol. 3, no. 1, pp. 95-101, 2007.

[19] R. Balaji Raja and P. Singh, "Synergistic effect of silver nanoparticles with the cephalexin antibiotic against the test strains," Bioresearch Bulletin, vol. 2, no. 4, pp. 171-179, 2012.

[20] I. N. Ghosh, S. D. Patil, T. K. Sharma, S. K. Srivastava, R. Pathania, and N. K. Navani, "Synergistic action of cinnamaldehyde with silver nanoparticles against spore-forming bacteria: a case for judicious use of silver nanoparticles for antibacterial applications," International Journal of Nanomedicine, vol. 8, pp. 4721-4731, 2013.

[21] K. I. Batarseh, "Anomaly and correlation of killing in the therapeutic properties of siliver (I) chelation with glutamic and tartaric acids," Journal of Antimicrobial Chemotherapy, vol. 54, no. 2, pp. 546-548, 2004.

[22] A. Contreras and D. Vazquez, "Cooperative and antagonistic interactions of peptidyl tRNA and antibiotics with bacterial ribosomes," European Journal of Biochemistry, vol. 74, no. 3, pp. 539-547, 1977.
[23] S. Saha, M. M. Malik, and M. S. Qureshi, "Comparative study of synergistic effects of antibiotics with triangular shaped silver nanoparticles, synthesized using UV-light irradiation, on Staphylococcus aureus and Pseudomonas aeruginosa," Journal of Biomaterials and Nanobiotechnology, vol. 5, no. 3, pp. 186-193, 2014. 

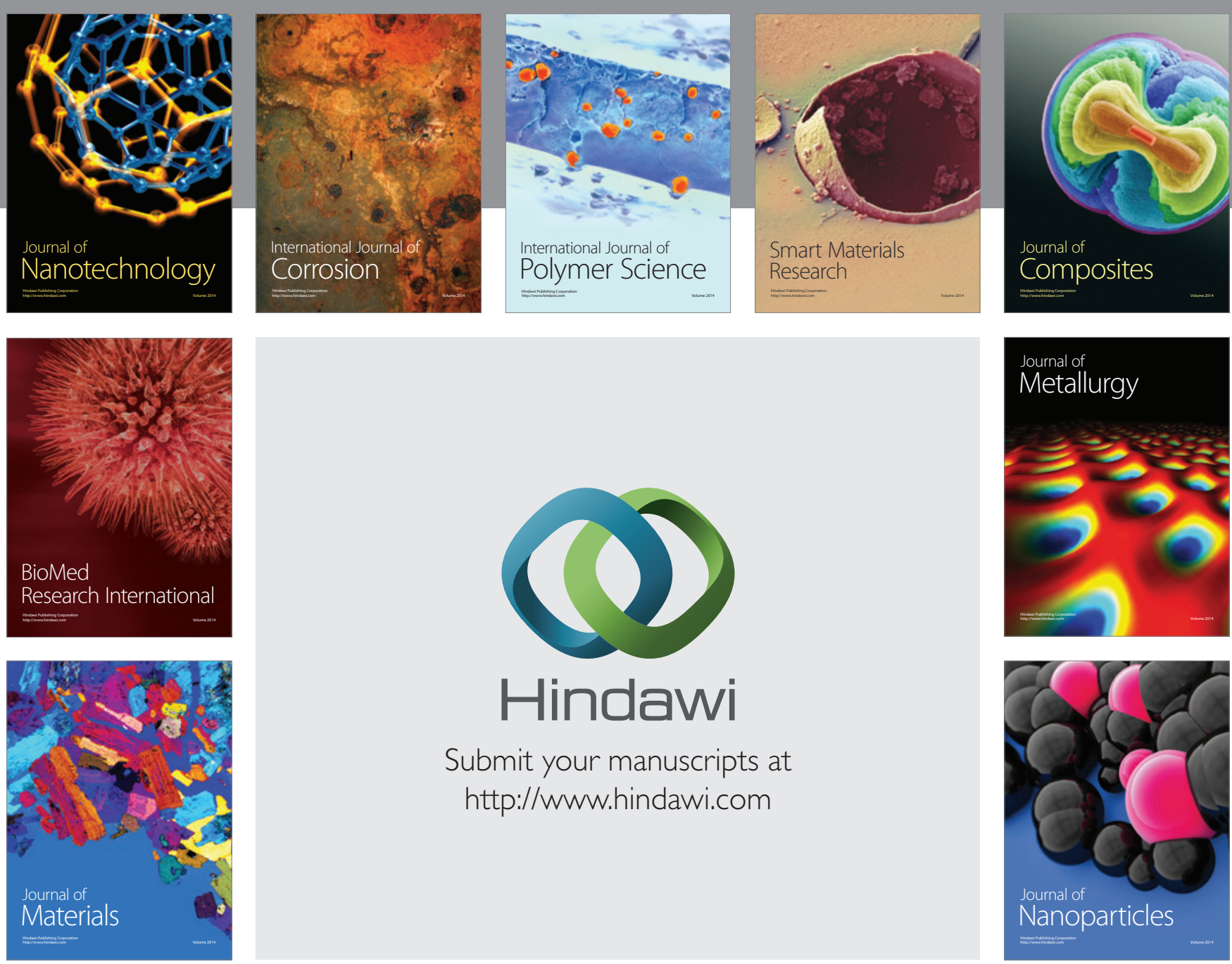

Submit your manuscripts at http://www.hindawi.com
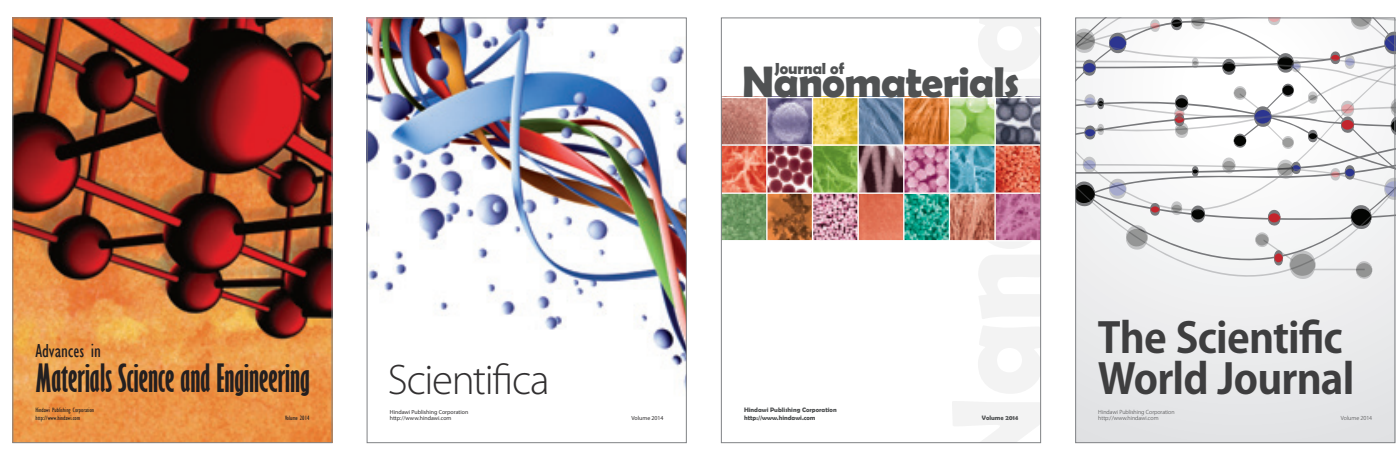

\section{The Scientific World Journal}
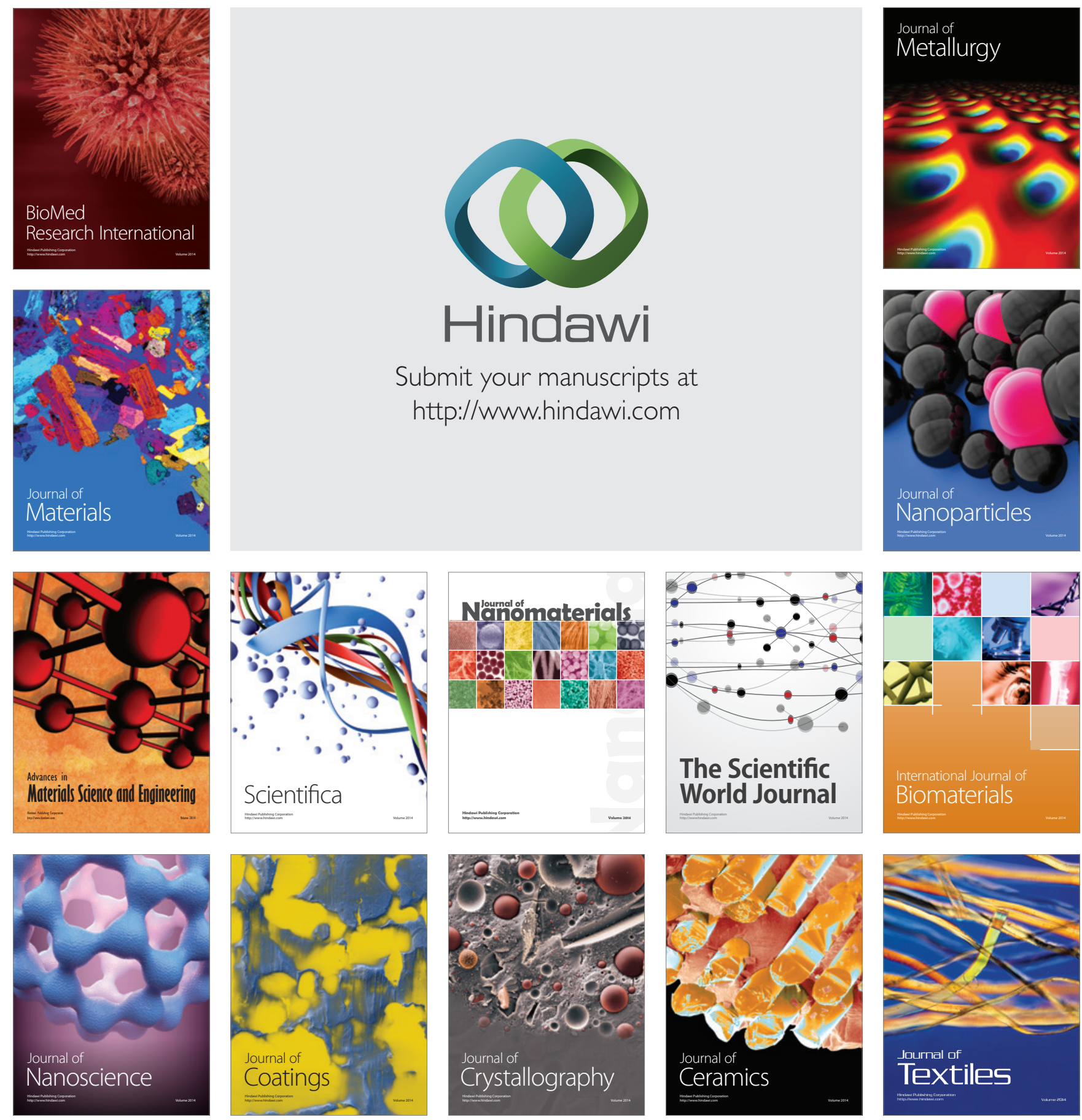